\title{
Entre redynamisation urbaine et banalisation des espaces : tensions et enjeux de l'urbanisme touristique
}

\section{Vincent Vles}

\section{(2) OpenEdition}

\section{Journals}

Édition électronique

URL : http://journals.openedition.org/tourisme/507

DOI : 10.4000/tourisme.507

ISSN : 2492-7503

\section{Éditeur}

Éditions touristiques européennes

\section{Édition imprimée}

Date de publication : 1 juin 2011

Pagination : 14-25

ISSN : 2109-5671

\section{Référence électronique}

Vincent Vles, «Entre redynamisation urbaine et banalisation des espaces : tensions et enjeux de I'urbanisme touristique », Mondes du Tourisme [En ligne], 3 | 2011, mis en ligne le 30 septembre 2015, consulté le 19 avril 2019. URL : http://journals.openedition.org/tourisme/507 ; DOI : 10.4000/ tourisme.507

\section{(c) $(1) \&$}

Mondes du tourisme est mis à disposition selon les termes de la licence Creative Commons Attribution - Pas d'Utilisation Commerciale - Pas de Modification 4.0 International. 


\title{
Entre redynamisation urbaine et banalisation des espaces : tensions et enjeux
}

\section{de l'urbanisme touristique}

\author{
VINCENT VLÈs_[vincent.vles@univ-pauff] \\ Professeur d'aménagement et d'urbanisme - Université de Pau et \\ des pays de l'Adour
}

Résumé. Dans une société devenue celle de l'image

et de la notoriété, les urbanistes des villes et des

stations transforment les espaces publics urbains

emblématiques de manière à permettre au touriste

d'en saisir l'originalité et l'harmonie. En mettant en

scène le décor, ils cherchent à privilégier les expériences,

les sensations, les rencontres, et à appréhender le

visiteur comme un nouvel acteur de la vie urbaine,

acteur qui participe à la fabrication narrative de la

cité. Cependant, les excès du tourisme de masse

provoquent souvent une banalisation de ces espaces

emblématiques, confrontés aux questions des conflits

d'usage et d'appropriation.
Abstract. Town planners transform the emblematic public spaces of cities and tourist resorts in order to favour their image and their notoriety. They try to allow tourists to get their originality and harmony. Therefore, town planning privileges great build-up with experiments, feelings, meetings; it regards the visitor as a new actor of urban life, an actor who takes part in the narrative construction of the city. However, mass tourism often robs emblematic spaces of its authenticity, creating problems like conflicts of use and appropriation. 
n quoi l'urbanisme per-
met-il d'améliorer l'image
d'une ville ou d'une station? La question peut sembler naïve. Il n'est peut-être pas vain d'en rappeler l'importance pour l'élaboration du projet participatif de ville ou de station. L'une des particularités du tourisme en ville est qu'il se pratique essentiellement par la déambulation dans des espaces publics (places, rues, ruelles, traboules, terrasses, esplanades, jardins, cours...) : $70 \%$ des touristes urbains déclaraient en 2007 avoir fait une promenade en ville pendant leur séjour, $54 \%$ visitent la ville à pied (Odit France, 2008). Partie du domaine public non bâti, affectée à des usages publics, l'espace public des urbanistes est formé par une propriété et par une affectation d'usage (Merlin et Choay, I996, p. 334). Concept d'essence polysémique et transdisciplinaire, il relève à la fois de la sphère politique (Habermas, 1978), sociologique (Goffman, 1973; Germain, 2002, pp. 25-3I) et spatiale (Toussaint et Zimmermann, 200I). L'approche urbanistique et architecturale l'appréhende dans tous ses aspects : "L'espace public renvoie à une totalité, celle de la ville, et à la diversité de ses quartiers, de ses fragments. Il fait corps avec un lieu, des architectures qui lui donnent des fonds, des couleurs, des fermetures ou des ouvertures. Il s'ouvre à des habitués, des étrangers, des enfants, des vébicules. Au niveau du sol comme au niveau du ciel, il croise la mémoire collective et l'usage individuel. Il nécessite enfin la collaboration d'un grand nombre d'intervenants - ce qui rend sa réalisation très complexe” (Picon-Lefebvre, 1997, p. 10).

\section{RESTRUCTURATION \\ ET MISE EN SCÈNE DES ESPACES PUBLICS}

La récente restructuration et la mise en scène des espaces publics à des fins récréatives dans les grandes villes témoignent des modifications qui ont transformé temps de travail et de loisirs, mobilité et sédentarité. Lieux de vie, d'événement, d'expression de l'identité et de l'ambiance, voire de "l'âme" d'un système urbain, parfois véritables agoras ou au contraire simples voies de cheminement et de passage, les places, rues, parvis, points de repère créent dans les villes des endroits privilégiés et libres d'accès où se mêlent l'habitant et le voyageur. Points de convergence où se rencontrent différents usages, de tensions sociales parfois, ils forment la partie la plus exposée de la mise en œuvre des politiques urbaines : lieux de connexion de projets de ville, ils régissent l'orientation des flux, deviennent vecteurs d'image et acquièrent ainsi une fonction stratégique de première importance (Bassand, 200 I, p. 23).

\section{Une image profondément renouvelée}

L'urbanisme participe à la fabrication de l'image parfois profon- dément renouvelée d'une ville, dont l'ouverture au tourisme contribue à accroître la notoriété - les quelques exemples convoqués par la recherche entreprise (Barcelone, Berlin, Lyon, Montpellier, Bordeaux, Marseille) sont symptomatiques d'un phénomène devenu aujourd'hui mondial. L'aménagement urbain y produit ou promeut un décor et une symbolique valorisant l'identité, le renom, l'attrait de la cité (Vlès, 2004, pp. I77186). Les architectes et les paysagistes ne gèrent plus l'espace public urbain seulement en termes de circulation des hommes et des réseaux : ils transforment sa capacité à créer une représentation d'activités sociales, du "vivre ensemble". Par exemple, la ville de Montpellier a compris très tôt l'effet bénéfique pour l'attractivité d'un positionnement fondé sur les loisirs (Fricau, 2008) : dès les années 1970, les pratiques récréatives, affirmées avec force comme véhicules d'une image valorisante, constituent l'un des cinq axes prioritaires de la stratégie de "technopolisation" à outrance d'une ville affichée comme surdonée. La construction de cette image, épaulée par un mouvement de décentralisation tombé à point nommé, a donné des résultats évidents que d'autres cités, de taille souvent moindre, ont tenté de reproduire, avec plus ou moins de rapidité ou de bonheur. La requalification et l'adaptation des espaces publics à la modernité sont devenues les outils privilégiés de la reconquête 
des villes ou de la lutte contre leur obsolescence. Les travaux des chercheurs concordent tous sur ce point : Rachel Rodrigues-Malta (2005) montre comment, dans un échantillon de plus de 73 villes portuaires européennes, plus de 50 opérations visent à réintégrer les fleuves, fronts de mer, docks, bref l'élément aquatique dans l'organisation territoriale et le fonctionnement urbain. Partout dans le monde, les friches artisanales, industrielles ou militaires sont restructurées au profit des activités ludiques, récréatives, commerciales, dans le but avoué d'accroître l'attractivité des centres-villes ; des cas aussi différents que l'aménagement de Bercy Village, la réhabilitation muséographique de la Jahrhunderthalle de Bochum, la construction du musée d'Art contemporain dans le quartier $\mathrm{El}$ Raval de Barcelone, le musée Guggenheim à Bilbao... sont en cela emblématiques (Vlès et al., 2005, p. 38). La qualité de l'image mentale que le voyageur se fait d'un quartier, donc a fortiori d'une ville, est directement liée aux liens qui unissent entre eux ces lieux à forte notoriété : les recherches publiées dans ce domaine ont montré que plus les espaces publics sont pensés, organisés, travaillés, plus la compréhension générale de l'ensemble est identifiable pour l'usager et son attractivité, meilleure (Pinon, 1991 ; Toussaint et Zimmermann, 2001 ; Odit, 2008 ; Park, 2008).

Cependant, compréhension ne signifie pas homogénéité ou bana- lisation, au sens de la réduction de la complexité de l'espace urbain : l'agencement de la place aux Huiles en liaison avec le cours Estienned'Orves, derrière le Vieux-Port de Marseille, crée judicieusement un espace calme, un "salon urbain", sans voitures, une scène propice à la rencontre, à l'échange qui rompt radicalement avec l'agitation alentour. Cet aménagement conduit très vite à une mutation des fonctions urbaines : les restaurants remplacent les entrepôts et les imprimeries, les voitures disparaissent en sous-sol et le vide, la lumière, l'espace délivrent le passant du plein et de l'oppression (Fricau, 2008, p. 237).

Les politiques publiques tentent ainsi de sauver les villes touristiques que le cadre bâti vieillissant condamne à la relégation au rang des sites connotés comme surannés, désuets, et ne pouvant s'adresser désormais qu'à un tourisme marginal, hors des lieux de la modernité et du progrès. Bien entendu, ce traitement ne vaut que pour son affichage symbolique : l'urbaniste ne peut travailler que sur des morceaux de ville et c'est de la cohérence de l'ensemble des quartiers que dépend l'émanation d'une ambiance urbaine. Car si les campagnes de promotion touristique peuvent réduire l'image de Bordeaux aux quartiers du "triangle d'or", de Saint-Pierre et aux quais réaménagés, celle de Marseille au VieuxPort ou à la Belle de Mai, celle de Montpellier à l'Écusson et à
Antigone, le voyageur ne s'y trompe guère et retient généralement un ensemble (Vlès et al., 2005). Il découvre des jeux de volumes entre les pleins et les vides, des "portes" qui délimitent une scène propice à la rencontre, à l'échange rompant parfois radicalement avec l'agitation alentour. Par cette rupture avec les quartiers environnants, le parti d'aménagement permet une respiration à la vie urbaine et à sa découverte. Cela conduit très vite à une mutation des fonctions qui lui étaient attribuées : les restaurants ont remplacé les entrepôts et les imprimeries, les voitures ont disparu en sous-sol et le vide, la lumière, l'espace ont remplacé le plein et l'oppression. C'est sur cette base que la transformation de bâtiments productifs en équipements ouverts au public, culturels ou récréatifs est un facteur constitutif du tourisme urbain, mais aussi, comme pour ces anciens arsenaux marseillais transformés en musée, elle contribue à la cohérence de l'image au profit des usages ludiques fondés sur ce qui est censé représenter (aux yeux du voyageur) l'essence de la vie locale, ici méditerranéenne.

Cet urbanisme "sensible" prend en compte les capacités de charge et les seuils de saturation, fabrique des ambiances, crée des émotions et les rend accessibles et perceptibles: il engage l'espace social urbain dans un rapport au monde, il se montre. À Montpellier, la place de la Comédie joue à la fois le rôle de parvis pour l'opéra, d'espace de 
rendez-vous autour de la fontaine, de porte d'entrée pour le centre commercial du Polygone - et, par extension, d'invitation à poursuivre vers Antigone -, de passage vers la gare, l'Écusson (centre historique) et vers l'Esplanade, qui la relie au siège du conseil régional. Les aménagements qui y ont été programmés en font "un seuil, un sas, un lieu où il faut passer pour entrer et saisir le sens de la ville. [Cette place] raconte l'exceptionnel, dit le banal, proment le trivial, permet le marginal" (Ferras et Volle, 2002, p. 155). Son positionnement, ses fonctions et son aménagement favorisent le mouvement : son agencement rectangulaire produit un effet de perspective qui appelle le passant à la traverser dans son axe le plus long, la quasi-absence d'obstacles en son centre facilite la déambulation et la transforme en promenade, les seules ouvertures visuelles en ses quatre coins la font lien entre l'Esplanade, le Polygone, l'Écusson, la gare. L'aménagement de la place de la Comédie, haut lieu théâtralisé de la vie urbaine, ne fournit pas seulement un cadre, il constitue la ressource première de son activité récréative en produisant une dynamique, en imposant un rythme, en impulsant des mouvements et des pratiques. Les choix d'urbanisme (et leurs corollaires en termes d'ambiance : acoustique, mobilité, environnement lumineux, olfactif, thermique, dimension esthétique) s'imposent ainsi aux politiques touristiques.

\section{L'urbanisme participe à la fabrication narrative de la cité}

Les urbanistes sont donc conduits à composer des espaces publics emblématiques, de manière à permettre à l'usager d'en saisir tant l'originalité et l'harmonie que le mode d'emploi dans une société qui est devenue celle de l'image et de la notoriété. Les plus grands architectes sont convoqués pour redessiner le décor de ces lieux dont on attend un rayonnement international. Pour l'habitant, mais aussi pour l'étranger, le visiteur, ambassadeurs potentiels devenus vecteurs de renommée, les places et les avenues sont transformées en lieux composites où se superposent des fonctions parfois contradictoires et des publics aux attentes antagonistes. En veillant à ce que soient satisfaits à la fois les usages économiques essentiels à la ville (services, commerces, bureaux, artisanat), la spontanéité des relations sociales (rencontres fortuites, échanges de plaisirs et de consommation touristiques, attente, flânerie, culture, spectacles, jeux...), l'attente des surprises sensorielles liées à la découverte de formes, d'événements nouveaux qui émergent des lieux de mémoire réhabilités ou nouvellement édifiés (le Mémorial de l'Holocauste de Berlin en constitue peut-être l'archétype contemporain), l'urbaniste réussit souvent, dans les villes étudiées, à introduire un imaginaire original (Antigone à Montpellier, la place aux Huiles à Marseille, El Raval à Barcelone, l'aménagement des quais de Bordeaux...) à nul autre lieu pareil, qui combatte l'ennui, appelle au souvenir, à la rêverie, à la promenade, à la tranquillité ou qui, au contraire, invite au mouvement, à l'exploration, à l'activité ludique, aux recours symboliques.

La fabrication narrative de la ville pour les touristes par l'aménagement de ses espaces publics est possible et a été mise en œuvre avec succès dans un certain nombre de cités ou de stations. Les travaux de recherche conduits depuis 2004 montrent comment le traitement urbanistique du cadre architectural et du bâti public, l'analyse symbolique des bâtiments et des fonctions produite pour le visiteur par l'architecture, leur recomposition par les pouvoirs locaux dans un "récit" révélant l'esprit du lieu (un agencement, une lecture paysagère, une interprétation proposée au passant $^{(1)}$ ) assurent à une stratégie de rayonnement de la ville un grand potentiel d'efficacité en tant que destination touristique et la démarquent de ses concurrentes. Le cas de Bordeaux est flagrant (Vlès et al., 2005 ; Fricau, 2008) : ici, de grands programmes d'architecture ont permis, à l'opposé du mimétisme, d'importer un "imaginaire au cour de l'ordinaire” (Urbain, 1998) et de donner au visiteur au moins l'illusion de s'affranchir du quotidien, dans le lieu même du quotidien. En la matière, l'influence de la pensée architecturale de l'école du 
Bauhaus, pour laquelle construire et montrer est avant tout une activité sociale, intellectuelle et symbolique, est considérable dans ses implications touristiques (Droste, 2002). Représenté par les réalisations de Walter Gropius et de Ludwig Mies van der Rohe, à Berlin, à Chicago ou à New York, le design planning du Bauhaus, né à l'avant-garde de la nouvelle construction des années 1920 à Weimar, a exercé une influence décisive sur la modernisation radicale de la vie et de ses phénomènes secondaires positifs et négatifs, d'autant que quelques-uns des plus éminents professeurs du Bauhaus ont acquis une influence grandissante hors d'Allemagne, surtout aux États-Unis. Aujourd'hui encore, la pensée du Bauhaus exerce ses effets formels dans la production de la ville contemporaine mondiale, surtout dans la manière dont il en détermine les rythmes, les formes et les couleurs qui la transforment en objet de contemplation. À l'affût de la nouveauté, de la singularité du quartier, l'urbanisme met ainsi en scène les découvertes (l'accès à la visite des traboules du vieux Lyon, les puits de la mémoire des autodafés nazis creusés dans la Bebelplatz en enfilade de l'avenue Unter den Linden de Berlin...), privilégie les expériences, les sensations, les rencontres. On y traite le visiteur non plus comme un étranger, cet individu autrefois considéré avec indifférence, mais comme un nouvel acteur de la vie urbaine. Imaginaire, perception des sens, expérience du visiteur sont sollicités dans ce qui est avant tout une rencontre. Cette attention nouvelle, qui fait du touriste un acteur de l'espace public urbain, transforme la ville en lieu soumis à une appropriation constante, toujours en mutation. Son aménagement relève autant du rationnel et du sensible, de l'expérience subjective et de la perception que du fonctionnel. La place de la Comédie à Montpellier, le Mémorial de l'Holocauste ou la porte de Brandebourg à Berlin, la place PeyBerland à Bordeaux, le cours Estienne-d'Orves à Marseille ont été métamorphosés par un simple aménagement de surface et par un choix réfléchi des matériaux et des couleurs pour les sols mettant en valeur l'architecture périphérique, la théâtralité du décor et sa large ouverture à toutes les strates de la société.

Cet autre regard, qui fait du touriste un habitant et de l'habitant un touriste, en tout cas un acteur de la mise en scène de l'espace public urbain, transforme la ville en lieu ouvert à une appropriation constante, parfois conflictuelle, jamais définitive, un lieu d'orientation, de bifurcation, d'attente, de déplacement. Son aménagement relève plus du rationnel et du sensible, de l'expérience subjective et de la perception que du fonctionnel. Le traitement des façades y est essentiel, mais souvent l'aménagement du sol suffit à produire l'am- biance d'un lieu : le touriste apprécie positivement l'aménagement de la place de la Comédie à Montpellier, de Pey-Berland à Bordeaux, du cours Estienne-d'Orves à Marseille, qui ont été métamorphosés par un simple aménagement de surface mettant en valeur l'architecture périphérique, la théâtralité du décor par un choix réfléchi des matériaux et des couleurs pour les sols (Fricau, 2008).

\section{La question \\ de la rencontre favorisée entre visiteurs et habitants n'est pas tranchée}

L'ambiance semble déterminée par l'action qui s'y déroule (Vlès et al., 2005). Un décor sans intrigue, sans action est comme une scène vide. Sa mise en scène ne peut être artificielle : le recours systématique à un événement éphémère ne suffit pas à produire une ambiance ordinaire. Elle dépend de l'appropriation des lieux par les populations. Sa "mise en tourisme" implique donc concrètement de dramatiser le lieu, de le mettre en perspective, de s'affranchir de la seule représentation utilitaire et fonctionnelle (Joseph, 1995). C'est ce que montrent ces nouveaux îlots contemporains de bureaux construits depuis les années 1990 à Berlin, à Bordeaux, insérés parfois brutalement dans des quartiers XVIII ${ }^{e}$ très homogènes (la Cité mondiale dans les Chartrons, par exemple). Avec ses façades en verre dans un quartier de pierre, ses arrondis dans un 
espace très rectiligne, sa hauteur graduée émergeant du secteur bâti en " $\mathrm{R}+3$ ", de tels îlots tempèrent, par effet de contraste, l'image monotone du quartier environnant. De même, la création de la place des Pistoles dans le "Panier" à Marseille, au croisement de quatre lieux touristiques (la cathédrale de la Major, la rue de la République, la Vieille Charité et le Vieux-Port), octroie au patrimoine local une nouvelle polarité, en créant une attraction. "Entourée d'un grand nombre de monuments historiques, la place ainsi restructurée accueille des animations traditionnelles tout en créant un nouveau parvis dont la force jaillit grâce au recul, structuré par des banquettes filantes s'ordonnant autour d'une fontaine, un rattrapage des niveaux par une série d'emmarchements et de murets de soutènement qui invitent à la découverte et à la déambulation, l'implantation d'arbres pour le jeu d'ombres et de lumières et d'appareils d'éclairage pour y prolonger la vie en nocturne. L'édification d'une fontaine et d'un mur d'eau redonnent un sens à la toponymie (rue du puits) et recréent une ambiance de place méditerranéenne" (Fricau, 2008, p. 178). Cette place, à caractère récréatif pour les jeux qu'elle incite auprès des habitants, est également touristique par le patrimoine qu'elle met en scène. Cette double fonction favorise la rencontre entre les voyageurs et la société locale.

L'embellissement du quartier de
Vésone à Périgueux montre, $a$ contrario, les limites d'une greffe mal traitée. Confiée à Jean Nouvel, la réalisation sans ancrage spatial ou temporel d'un musée dans ce quartier historique (bâti autour de ruines romaines), totalement laissé à l'abandon dans un paysage urbain sans intérêt ni structure, pose avec acuité le problème de la contemporanéité des principaux espaces publics touristiques. La construction et la diffusion de la nouvelle “image de marque" de ce site n'ont pas été rendues possibles parce que le traitement global de son environnement immédiat est resté en friche. La représentation que s'en font l'habitant et le voyageur est influencée par l'expression du quartier, sa représentation sociale, sa fonction, son histoire et même son nom. Le rôle de l'urbanisme touristique, qui est de mettre à jour, de modifier, de rendre lisible ou de renforcer cette signification, n'a pu être tenu (Vlès, 2002).

\section{TENSIONS LIÉES}

\section{À LA MISE EN TOURISME DES VILLES}

Cependant, les stratégies qui placent les villes touristiques en concurrence dans un contexte économique incertain condamnent leurs centres à se démarquer parfois à outrance et de manière très artificielle. Certaines en arrivent à se couper de ce que souhaitent leurs habitants et à tourner le dos aux racines culturelles qui ont façonné leur iden- tité. On retrouve ici cette particularité du tourisme qui tend à détruire ce qui le construit lorsque les foules, lasses d'une rencontre qui pourrait se passer ailleurs, presque n'importe où désormais, piétinent dans des lieux où l'identité, l'authenticité ont été gommées. Si les recherches engagées ont décelé ces tensions dans l'ensemble des villes étudiées, elles apparaissent plus amplement à Barcelone, sans doute en raison de la très grande notoriété de cette métropole qui s'est orientée vers le tourisme de masse depuis la décennie 1980 .

\section{Tensions autour de}

\section{l'appropriation de l'espace public}

La qualité et l'identité d'une ville tiennent avant tout à son ambiance ordinaire : elles sont acquises dès lors que ses hauts lieux demeurent fréquentés par les habitants, que les usages y sont pluriels, que la mixité sociale s'y maintient. Ce partage de l'espace est très vite confronté aux questions de l'appropriation des espaces visités et aux conflits d'usage. Par exemple, la politique touristique suivie à Barcelone depuis une douzaine d'années montre des signes de débordement évidents (Vlès et al., 2005, p. 66). Fait remarquable et rare, elle y avait acquis une grande efficacité grâce à l'action d'un organisme de coordination associant le secteur privé et le secteur public, qui reposait sur un consensus social initialement largement partagé. Barcelone a été une des premières 
villes au monde à veiller à toujours articuler l'image touristique avec l'aménagement urbain, la morphologie de l'espace devant faire écho aux grands thèmes structurants du récit de la ville, et inversement : "La revaloritzacío de la ciutat $i$ de l'espai públic com a seguretat $i$ ciutadania” (Borja, 200 I, p. 100). Hélas, poussée par les grands événements liés à la mondialisation touristique (tels les Jeux olympiques, le Forum universel des cultures de 2004, les opérations du type 22@ou Diagonal Mar, qui ont doublé le nombre de lits dans les hôtels en dix ans), une nouvelle politique touristique est apparue au grand jour en 1993, avec la création de Turisme de Barcelona, organisme mixte issu de la municipalité en collaboration avec la chambre de commerce et d'industrie et avec la Fondation pour la promotion internationale de Barcelone. Cet organisme a facilité l'inscription du tourisme comme un des axes fondamentaux du développement de Barcelone : le Plan stratégique de la ville, dès 1993, en fait effectivement une de ses priorités. C'est ainsi que l'urbanisme s'est vu grandement subordonné à cet objectif (Ajuntament de Barcelona, 1993). Du coup, les idées qui inspiraient ce modèle barcelonais existent toujours, mais elles ne se concrétisent plus que dans la réalisation ou la rénovation de quelques interventions dont le rôle est devenu très secondaire, sinon marginal, subordonné à une autre politique, celle des opérations immobilières majeures. La Barcelone post-olympique cherche à rentabiliser les investissements considérables qui y ont été programmés, y compris dans l'extension de l'espace public.

Prétendant au rôle de place internationale, Barcelone n'a eu de cesse de faire écho aux modes en matière de notoriété urbaine. Se voulant "globale", la ville est entrée dans un système concurrentiel et s'est engouffrée dans un partenariat avec le secteur privé où celui-ci impose ses propres règles de rentabilité liées au système financier international. L'effet urbanistique saute aux yeux avec les opérations du type 22@ou Diagonal Mar, où triomphe un urbanisme frontal, mondialisé, sécrétant une ségrégation sociale aiguë et un paysage de centres commerciaux géants sans rapport aucun avec l'image millénaire ciselée par les micro-aménagements des quartiers de l'hypercentre. Le Forum mondial des cultures 2004, qui se voulait un substitut, en termes de mobilisation socio-économique, aux Jeux olympiques, confirme cette politique d'urbanisme touristique banalisée. C'est un choix qui n'a en aucune sorte été prescrit par le touriste. Au contraire, Berlin a investi, pour son gigantesque quartier historique Museuminsel, dans un aménagement soucieux du respect de l'art et du passé prussiens - "eine grossartige Vision" ["une vision grandiose"] selon l'hebdomadaire Welt am Sonntag. Ici, on s'est situé à l'opposé de la réduction narrative, à l'opposé du décor planté qui impose une image finalement factice, réduite, fabriquée de toutes pièces, à l'opposé d'une mise en scène déconnectée des racines identitaires du lieu. À Barcelone, le récit fait de la métropole méditerranéenne par l'aménagement urbain depuis une dizaine d'années, très simplifié, a tendance à réduire l'histoire de la ville racontée aux passants par le mobilier urbain à quelques rares moments, concentrés sur les lieux de notoriété touristique (Vlès et al., 2005, pp. 64-74). Comme si le voyageur devait se limiter au quartier gothique, aux Ramblas, à la Barceloneta, à Montjuich, à la Sagrada Familia et, éventuellement, au Tibidabo, à Pedralbes, à l'Eixample. Il est vrai que, sur ces quelques espaces ouverts au public et singulièrement au voyageur, on compte aujourd'hui plus de 4 millions de touristes par an. Cette évolution vers le tourisme de masse renforce ainsi une image réductrice de la ville. Elle crée des externalités négatives, que l'équipe de recherche a observées au sein ou à propos de l'espace public.

\section{Réduction narrative du paysage urbain}

De grandes cités, mais également plusieurs villes stations (notamment sur les littoraux), se sont ainsi engagées dans d'inquiétants processus de réduction narrative de leur héritage patrimonial qui, s'il permettent de répondre immédiatement aux 
exigences du tourisme de masse, entraînent également des effets négatifs dont l'intensité, parce qu'elle est contraire aux principes du tourisme durable, conduit à réévaluer la politique qui le soustend. Toutefois, la question des implications de cette fabrication narrative n'est pas simple à trancher. Car les effets de mode et la mondialisation ont produit des villes où se côtoient souvent conformisme et banalité : mêmes mobiliers urbains, systèmes et signalétiques de circulation normés, structures paysagères reproduites à l'infini, absence d'identité et d'originalité. L'urbaniste a offert souvent au tourisme de masse et à ses grandes migrations des destinations banalisées, aux différences gommées ; les places, les rues des villes et des stations sont ainsi devenues stéréotypées et ont été insérées dans un mode de consommation uniformisé, bâti autour d'avenues ou de dalles dans lesquelles s'imbriquent des centres commerciaux. Le constat n'est pas nouveau : le tourisme banalise et, dès les années 1890, Paul Jousset écrivait : " $\mathrm{La}$ banalité, gagnant de proche en proche, a répandu sur toutes choses sa teinte grise uniforme. Plus de couleur ; le pittoresque disparaît : toute la France se ressemble" (Jousset, 1893). On retrouve dans la généralisation de la production urbaine ces ruptures d'ancrage temporel des villes qui, en emportant toutes les différences, enlèvent son sens au voyage. Maintenant averti, le touriste assimile toujours plus les différences. À Barcelone comme ailleurs, aujourd'hui, les villes reviennent sur une architecture “années 1970" qui faisait coller, à l'époque, l'adaptation du cadre bâti aux attentes du "client" : en suivant cet aménagement standardisé, "moderne", elles ont couru le risque de se démoder dès l'avènement du postmodernisme. Car "la demande" est par nature éphémère. Certes, on constate, au cours des entretiens, que le touriste demande des besoins qu'on lui suggère, mais, exigeant, il a acquis au fil de ses expériences une capacité de décision et de mobilité qui déroute en permanence les professionnels. Les entretiens que nous avons réalisés montrent que, si le touriste fréquente les lieux commerciaux avec assiduité, il semble peu apprécier le style banalisé. D’autant que le cliché, le stéréotype, la pauvreté architecturale ont façonné des quartiers peu propices à redécouvrir ce qui fait l'authenticité de la ville. À Barcelone comme ailleurs, ils sont le fait d'une architecture de marketing largement programmée dans les opérations de rénovation ou de requalification urbaines, soumises aux concepts réducteurs du quartier commercial des chaînes franchisées plutôt qu'à la recherche, par le voyageur, de lieux de convivialité, de sociabilité, d'échanges culturels.

Le paysage urbain que cherche à projeter Turisme de Barcelona constitue aujourd'hui une entrée incontournable pour comprendre le processus de réduction narrative dans lequel la ville est entrée. En effet, les récits que sous-tendent les aménagements publics (traitements paysagers des dalles par un mobilier urbain futuriste - lampadaires effilés, malles corsaires en fonte, bancs à une place...) cherchent à imposer, sans ambiguité, l'image d'une ville dynamique et contemporaine à tous points de vue. Le passé y est valorisé seulement par le rappel systématique de "deux époques de splendeur" : l'époque "gothique" et celle $\mathrm{du}$ "modernisme". Ces deux époques ont certes toutes deux laissé une empreinte remarquable dans la morphologie de la ville et de ses espaces publics. Mais cette réduction de l'histoire de la ville à deux époques laisse un champ peutêtre trop important à l'élan créatif et international de la Barcelone contemporaine : elle applique la même rhétorique à l'embellissement du paysage urbain ancien qu'à la construction ou restructuration des quartiers nouveaux.

De plus, des opérations comme le Forum des cultures ou le centre commercial de Diagonal Mar, réalisées dans les années 2000 pour donner une image de ville-monde, conduisent, en dépit de quelques immeubles originaux, à produire des imitations de paysages qu'on retrouve ailleurs, dans la plupart des villes. C'est un peu comme si la politique touristique avait encouragé un urbanisme appauvri, à l'image de son récit. 
À Barcelone, la scène s'est vidée par endroits de toute urbanité locale. L'espace public y a été manipulé pour satisfaire une image internationale, et non pour le citoyen. Est-ce source de conflits à venir ? Très probablement, car, comme l'exprime Jordi Borja : “ $\grave{A}$ Barcelone, la dynamique qui peut nous conduire à devenir un parc à thème mondialisé est forte, quoique les résistances des citadins soient aussi capables de renouveler l'urbanisme citoyen" (Borja, 200I, p. 100). Le cas de Barcelone montre comment le tourisme, lorsqu'il devient "de masse", engendre des codes et des usages spécifiques, à tendance conflictuelle. Les temps d'attente, la densité trop forte provoquent des tensions antagoniques qui finissent par nuire à l'image de la ville.

\section{Stratégies d'évitement de la part des habitants}

La production de cette image standardisée n'est pas sans effets sociaux. Les entretiens accompagnés ont montré les stratégies d'évitement mises en œuvre par les habitants dans leurs cheminements quotidiens, premier signe d'un conflit latent (Vlès et al., 2005, p. 75). Sur les fameuses Ramblas du centreville, le flot des touristes est tel que le Barcelonais a perdu l'habitude de s'y promener, sauf le dimanche - où il semble reprendre quelque peu possession de cet espace ; le reste du temps, l'habitant utilise d'autres voies plus ou moins paral- lèles. La rue Ferran, connue depuis longtemps comme axe commercial de qualité, en fournit le meilleur exemple; la multiplication récente des commerces et services visant le tourisme de masse ("fast food", magasins de vêtements, de souvenirs et d'autres articles bas de gamme) ont fait tellement chuter, notamment par leurs enseignes, l'esthétique de la rue, que l'habitant se surprend depuis un certain temps à ne plus l'emprunter, préférant plutôt les ruelles qui la longent. Ce phénomène de dégradation visuelle et d'évitement ne se rencontre pas qu'à Barcelone. Il se généralise dans toutes les villes et stations qui ne mettent pas en place un cahier des charges strict d'urbanisme commercial, surtout dans leurs parties centrales où les peintures et les néons aux couleurs criardes dégradent visuellement les espaces publics et les coupent de leur environnement patrimonial. Évitement et réduction narrative posent avec acuité la question de l'appropriation des lieux, du partage de l'espace, de la mixité des usages qui sont les conditions du maintien d'une ambiance ordinaire nécessaire tant à la vie sociale normale de la ville qu'à son attrait pour le voyageur, donc à son attractivité réelle et à son identité à long terme (Puca, 2008).

Le fait que la population commence à s'interroger - ainsi que nous avons pu le constater dans les entretiens que nous avons réalisés (Vlès et al., 2005) - sur la res- semblance entre les services, les commerces, les animations de rue indique une certaine prise de conscience de la transformation paysagère de la ville liée au tourisme de masse. À Barcelone, mais ce ne saurait être un cas d'espèce, même la presse se fait l'écho de ce que les Ramblas deviennent "le pire magasin du monde" (cf. El Periódico du 25 février 2005), n'offrant que des souvenirs bon marché et de mauvais goût (du "mannequin-pisse" en habit catalan au "sombrero mexicain") dans des magasins tenus par des Asiatiques. On constate également la gêne que le tourisme procure aux habitants en termes de bruit, de coûts de nettoyage et d'embouteillages (du fait, par exemple, de la noria des cars qui s'arrêtent Passeig de Sant Joan, comme à Berlin près du mur, au niveau du musée Haus am Checkpoint Charlie). Ces conflits demeurent pour la plupart latents, voire larvés. Même s'il est encore le fait d'une minorité intellectuelle, l'écho remarquable des questions urbanistiques au sein de la population risque de donner de l'ampleur aux enjeux ainsi soulevés. Les enquêtes menées en promenades accompagnées révèlent une ville parfois vécue comme un parc d'attractions ou parc à thèmes (à Barcelone, le terme "un parque temático" a été plusieurs fois prononcé). L'offre patrimoniale se trouve précisément là où bat le cœur de la ville, là où l'ambiance seule suffit à faire son charme. Or 
cette ambiance repose sur l'usage de l'espace par ses habitants, qui maintenant le désertent.

\section{Persistance ou accentuation des formes \\ de ségrégation urbaine}

Ce discours réducteur lié au tourisme de masse accentue également certaines formes de ségrégation. Ce sont d'abord les touristes qui se retrouvent complètement isolés. Cela est particulièrement visible dans le mobilier urbain et la signalétique nouvelle qui envahissent l'espace public, où l'anglais semble être le moyen d'atteindre le plus grand nombre. Ainsi, de plus en plus d'annonces ou de menus sont affichés exclusivement en anglais. Les affiches multilingues disparaissent progressivement. Dans ce contexte où, bien souvent, l'employé d'un musée, d'un restaurant ou d'un café s'adresse directement au touriste en anglais de préférence à sa langue natale, l'interaction culturelle entre la culture locale et celle du visiteur semble le dernier des soucis. L'invitation au dialogue n'est pas au rendez-vous. La ségrégation est clairement lisible. Et il est intéressant de voir combien celleci rebondit encore à propos de l'enjeu urbanistique. La stratégie de promotion d'une image simplifiée de la ville a conduit à privilégier le produit touristique constitué par quelques lieux précis et massivement, voire trop, fréquentés. De nombreux conflits urbains ont eu lieu dans les secteurs dont la popu- lation a été activement chassée (notamment à Poble Nou, dans le nord-est, en raison du plan 22@).

La muséification et la gentrification des centres urbains font également partie des effets pervers généralement constatés dans le cadre des cités à forte notoriété (Duhamel et Knafou, 2007, p. 18). La patrimonialisation excessive relevée à Venise constitue un cas d'école, que l'on retrouve jusqu'à San Francisco ou Montréal. Changement de pratiques habitantes, changement de population, mutation des commerces et arrivée des touristes figent l'espace par des procédures de protection et de mise en valeur. Les façades des bâtiments, les matériaux utilisés ou les modifications à l'intérieur du bâti sont réglementés pour éviter la dégradation du paysage urbain. Souvent abrités dans un secteur sauvegardé ou dans une zone de protection du patrimoine architectural et paysager, les quartiers historiques subissent des opérations de restauration visant à rendre salubres des habitats et des espaces dégradés. L'amélioration du parc de logements conduit à une hausse des prix de l'immobilier et attire de nouvelles populations, souvent plus aisées que celles qui sont en place. Le tourisme n'est pas directement à l'origine de ces effets pervers : même s'il contribue à une hausse des prix, il n'est ici que le vecteur indirect favorisant la mobilité des capitaux, un facteur d'accompagnement issu de la mobilité et de la mondialisation. Il est cepen- dant associé à ce mouvement qu'il renforce.

\section{Un risque avéré de vacuité \\ et de paysage urbain dépourvu de signes distinctifs}

De tout cela, on peut dégager quelques enseignements quant aux rapports entre urbanisme, habitant et tourisme. Un premier constat a trait à la ségrégation. D’un côté, la valorisation, en termes d'image, de certains ensembles patrimoniaux impose une lecture unique et, conséquemment, une politique d'aménagement qui exclut toute présence qui ne lui corresponde pas. De l'autre, la réduction narrative masque aux touristes des pans entiers de la ville et de sa culture, renforçant la pauvreté narrative de l'image communiquée. Autre constat, ce n'est pas seulement le touriste qui semble un peu perdu dans l'univers qu'on lui a mis en scène. C'est aussi souvent, à Bordeaux ou à Barcelone, le cas de l'habitant ! En effet, la rapidité avec laquelle l'espace public a été transformé et, surtout, avec laquelle une certaine image de la ville a été affichée, fait un peu de l'habitant un touriste dans sa propre ville. Intéressé par les travaux d'urbanisme et souvent flatté par l'ampleur des réalisations, il ne peut s'empêcher de s'interroger sur son propre rapport à son milieu de vie, à son propre passé dans la ville. D'un espace public limité ou sacrifié aux encombrements, il se retrouve dans des voies ou des sites le plus 
souvent piétonniers et d'une étendue dont il n'avait pas l'expérience.

L'analyse du fonctionnement des espaces publics urbains montre que le danger conflictuel lié au tourisme est pour l'instant limité à ce risque de vacuité et de banalisation. Ce risque apparaît lorsque les récits permis par les opérations d'urbanisme sont pauvres de sens, simplifiés à l'extrême et fondés sur la banalité et l'imitation. Les aménagements de l'espace public urbain participent réellement d'un argumentaire associé à l'image de la ville, donc hautement touristique, destiné à capter de nouveaux flux de visiteurs ou, plus simplement, à renouveler l'idée qu'on s'en fait ailleurs, à accroître son attractivité. Les traitements de la forme des espaces public urbains, les travaux sur leur composition, leur mise en lumière, leur ambiance sonore transforment ces espaces de rencontres et induisent des conflits, parfois réels mais la plupart du temps latents, entre tourisme et pratique quotidienne des habitants.

\section{ConClusion}

Le tourisme accompagne le processus d'appropriation ou de réappropriation de la ville : l'urbanisme en tient désormais le plus grand compte dans ses projets. Mais l'aménagement touristique est valorisant pour la ville s'il introduit de la contingence, des événements fortuits et imprévisibles, s'il préserve la liberté de construction narrative des habitants : se déplacer devient une invitation à la découverte et à l'inattendu si le touriste et l'habitant trouvent une ville surprenante par les œuvres qu'elle expose et les récits multiples et différents qu'elle leur donne à construire. Plus que l'image issue de mises en scène banalisées, la contingence des situations et des découvertes donne l'occasion de faire intervenir l'imaginaire au cœur de l'ordinaire, et permet de s'affranchir du quotidien. L'habitant devient dans cette optique non seulement bénéficiaire de la mise en tourisme des espaces publics urbains, mais il en est centre, le point de départ symbolique et social. Ce scénario semble être celui de la ville qui échappe à la muséification, qui fonctionne bien et contribue à gommer ce qui peut faire conflit entre habitants et touristes, ce qui renforce les satisfactions individuelles. La stratégie à suivre viserait alors à promouvoir des images complexes de la ville, à ne jamais privilégier un "produit" touristique constitué uniquement de quelques lieux précis, à passer de la gestion de sites isolés remarquables à des ouvertures aux touristes d'espaces et d'ambiances variés, socialement habités : équipements publics, petits commerces traditionnels, voies de types divers parfois séparées, parfois associées sur des parcours communs. L'aspect primordial des initiatives réussies de revitalisation des centres-villes touristiques réside bien dans cette intention de construire la ville " $\mathrm{du}$ et" ("die Stadt des und") et d'échapper à la ville exclusive ("die Stadt des entweder oder") "de l'un ou de l'autre" (Beck, 1996). Pour l'urbaniste, le récit à promouvoir, celui qui va sous-tendre l'image touristique de la ville, respecte et traduit l'assemblage des éléments divers qui font le vécu quotidien de la population résidente. Construire une politique de tourisme urbain n'est pas seulement un processus esthétique, c'est aussi une question d'organisation sociale et économique.

À une échelle très différente, les travaux de recherche en cours conduits par l'équipe dans des stations, françaises ou étrangères, littorales (La Grande-Motte, PortBarcarès, Seignosse, Saint-Jeande-Monts, Cambrils) ou en montagne (Gourette, Saint-Lary, la Pierre-Saint-Martin, Latas), vont maintenant vérifier comment ces problématiques d'un urbanisme en tensions s'y posent, notamment dans le cadre des expérimentations de modes de requalification plus ou moins réussis.

( I) Par exemple, l'expression symbolique par l'architecture de l'idée de transparence dans la cité démocratique : le tribunal de grande instance de Bordeaux (architecte : Rogers, 1999), le Reichstag de Berlin (architecte: Forster, 2000) ou la structure autoporteuse de la gare de transit aux abords immédiats de l'ancien World Trade Center de New York (architecte : Calatrava, 2009). 


\section{RECHERCHE • VARIA}

\section{RÉFÉRENCES BIBLIOGRAPHIQUES}

$\overline{\text { Ajuntament de Barcelona, }}$ Pla estratègic de turisme de Barcelona. Pla d'Accions, Cambra oficial de comerç, indústria i navegació de Barcelona, 1993.

Michel BASSAND, Anne COMPAgnON, Dominique JoYE et

Véronique SteIN, Vivre et créer l'espace public, Presses polytechniques et universitaires romandes, 200l.

Ulrich BECK, Die offene Stadt, DAB, 1996.

Jordi BORJA et Zaida MuXI, "L'espai públic : ciutat i ciutadania", Espai públic urbá, 2, Diputació de Barcelona, 2001.

Magdalena Droste, Bauhaus 1919-1933, Taschen, 2002.

Philippe Duhamel et Rémy KNAFOU, Mondes urbains du tourisme, Belin, 2007.

Robert FerRas et Jean-Paul VolLE, Montpellier Méditerranée,

Economica, 2002.

Baptiste Fricau, La Mise en scène à des fins touristiques des espaces publics urbains. Bordeaux, Marseille et Montpellier, thèse de doctorat en aménagement, Université de Pau et des pays de l'Adour, 2008.

Annick GermaIN, "La redécouverte de l'espace public : regards d'architectes et de sociologues", dans François THOMAS,

Espaces publics, architecture et urbanité de part et d'autre de l'Atlantique, Université de Saint-Étienne, 2002.

Erving GoffmaN, La Mise en scène de la vie quotidienne 2. Les relations en public, Les éditions de Minuit, 1973.

Jürgen Habermas, L'Espace public, Payot, 1978.

Isaac JOSEPH (dir.), Prendre place. Espace public et culture dramatique, Ministère de l'Équipement, Éditions Recherches-Colloque de Cerisy, 1995.

Paul Jousset, Un tour de Méditerranée, de Venise à Tunis par Athènes, Constantinople et Le Caire, Librairies-imprimeries réunies, 1893

Pierre MERLIN et Françoise ChOAY, Dictionnaire de l'urbanisme et de l'aménagement, Puf, 1996.

Odit France, Améliorer la qualité d'usage touristique des espaces publics en ville, coll. Mini-guides, Odit France, 2008.

Jungyoon PARK, Les Stratégies et projets urbains pour l'attractivité territoriale : rôle, place, et signification des nouveaux espaces de consommation urbaine, thèse d'aménagement-urbanisme, Institut d'urbanisme de Paris, 2008.
Pierre PINON, Lire et composer l'espace public, éditions du STU, 1991.

Virginie Picon-LefebVre (dir.), Les Espaces publics modernes. Situations et propositions, Le Moniteur, 1997.

PucA, L'Attractivité : maîtrise du temps et de l'espace, Actes du colloque international Puca (Plan urbain, construction architecture) - Paris 12 - Lille I, 2008 [en ligne].

À partir de www.urbanisme.developpementdurable.gouv.fr/puca [consulté les 17 et 18 novembre 2008].

Rachel RODRIGUES-MALTA, 'Villes, ports et 'waterfronts'. Expériences sud-européennes", Urbact working group SudestSustainable Development of Sea Towns Urbact programme, Naples Lead Partner, novembre 2005.

Jean-Yves Toussaint et Monique ZIMMERMANN, User, observer, programmer et fabriquer l'espace public, Presses polytechniques et universitaires romandes, 2001 .

Jean-Didier URBAIN, Secrets de voyage. Menteurs, imposteurs et autres voyageurs invisibles, Payot, 1998.

Vincent VLÈS, "Valorisation de l'imaginaire touristique du quartier de Vésone par l'aménagement des espaces publics", Ateliers et projets opérationnels du DESS Aménagement et gestion de stations touristiques, tome III, compte-rendu de recherche,

Université de Bordeaux 3, 2002.

Vincent VLĖS, "Espaces publics et mise en scène de la ville touristique", dans Vincent Berdoulay (dir.), L'Espace public à l'épreuve. Régressions et émergences, Maison des sciences de l'homme d'Aquitaine, 2004.

\section{Vincent Vlès (dir), Vincent Berdoulay, Sylvie Clarimont et}

Montserrat MORALÈs, Espaces publics et mise en scène de la ville touristique, rapport de recherche, Ministère délégué au Tourisme, direction du Tourisme et SET- CNRS - UPPA, 2005.

Vincent VLĖS, "Attractivité des villes touristiques : les limites du projet urbain", dans PuCA, L'Attractivité : maîtrise du temps et de l'espace, Ministère de l'Écologie, de l'Aménagement et du Développement durable, Plan urbain, construction, architecture, Paris 12, Lille I, novembre 2008. 